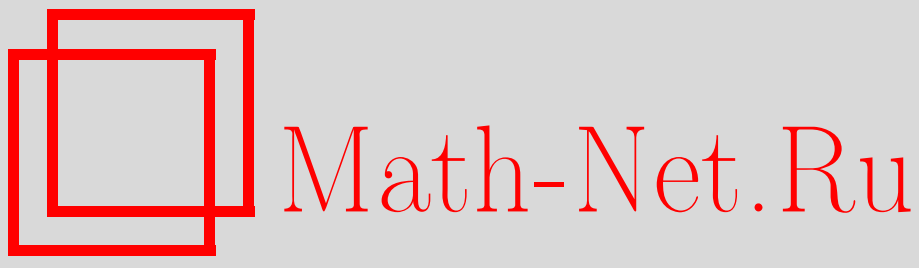

А. Буаве, П. М. Готье, П. В. Парамонов, О равномерной аппроксимации $n$ аналитическими функциями на замкнутых множествах в $\mathbb{C}$, Изв. РАН. Сер. матем., 2004, том 68, выпуск 3, 15-28

DOI: https://doi.org/10.4213/im484

Использование Общероссийского математического портала Math-Net.Ru подразумевает, что вы прочитали и согласны с пользовательским соглашением

http://www . mathnet.ru/rus/agreement

Параметры загрузки:

IP : 34.227 .88 .159

26 апреля 2023 г., 06:36:18 
УДК 517.538.5+517.956.2

\author{
А. Буаве, П. М. Готье, П. В. Парамонов
}

\title{
О равномерной аппроксимации $n$-аналитическими функциями на замкнутых множествах в $\mathbb{C}$
}

\footnotetext{
Приведены условия на замкнутое множество $F \subset \mathbb{C}$, необходимые и (или) достаточные для того, чтобы всякая функция $f$, непрерывная на $F$ и $n$-аналитическая на $F^{0}$, являлась равномерным на $F$ пределом некоторой последовательности $n$-аналитических целых или $n$-аналитических мероморфных функций.

Библиография: 15 наименований.
}

\section{$\S 1$. Введение}

Пусть $n \in \mathbb{N}$ - фиксированное натуральное число, и пусть $\bar{\partial}=\partial / \partial \bar{z}$ - оператор Коши-Римана. Функция $f$ называется $n$-аналитической (или полианалитической порядка $n$ ) на открытом множестве $G \subset \mathbb{C}$, если $f$ является $n$ раз непрерывно дифференцируемой $\left(f \in C^{n}(G)\right)$ и удовлетворяет условию $\bar{\partial}^{n} f=0$ в $G$. Нетрудно показать, что $f$ может быть записана в виде

$$
f(z)=f_{0}(z)+\bar{z} f_{1}(z)+\cdots+\bar{z}^{n-1} f_{n-1}(z)
$$

где $f_{0}, \ldots, f_{n-1}$ - голоморфные функции в $G$, причем указанное представление единственно.

Функция $f$ называется $n$-аналитической мероморфной в $G$, если ее можно представить в виде $(1)$ с функциями $f_{j}$, мероморфными в $G$. Если все функции $f_{j}$ из (1) одновременно являются полиномами или рациональными функциями комплексного переменного $z$, то $f$ называется $n$-аналитическим полиномом или $n$-аналитической рациональной функцией соответственно. Заметим, что $n$-аналитические рациональные функции не определяются как отношения $n$-аналитических полиномов при $n \geqslant 2$ и $n$-аналитические мероморфные функции могут быть непрерывны в своих особых точках, как, например, 3 -аналитическая мероморфная функция

$$
f(z)= \begin{cases}\frac{\bar{z}^{2}}{z}, & z \neq 0, \\ 0, & z=0,\end{cases}
$$

в точке $z=0$.

Работа первого и второго авторов выполнена при частичной поддержке грантами фонда NSERC (Канада). Работа третьего автора выполнена при частичной поддержке РФФИ (грант № 04-01-00720), программы Ведущие научные школы Российской Федерации (грант НШ-2040.2003.1) и фонда SAB (проект 98-0049, Испания). 
Пусть $F$ - замкнутое подмножество в $\mathbb{C}$ и $F^{\circ}$ - его внутренность. Через $C(F)$ обозначим пространство всех непрерьвных функций на $F$, а при $f \in C(F)$ положим $\|f\|_{F}=\sup _{z \in F}|f(z)|$. Введем классы функций

$$
\begin{aligned}
& \mathscr{A}_{n}(F)=\left\{f \in C(F) \cap C^{n}\left(F^{\circ}\right) \mid \bar{\partial}^{n} f=0 \text { на } F^{\circ}\right\}, \\
& \mathscr{E}_{n}(F)=\left\{f \in C(F) \mid \forall \varepsilon>0 \exists g \in \mathscr{A}_{n}(\mathbb{C}):\|f-g\|_{F}<\varepsilon\right\},
\end{aligned}
$$

а также $\mathscr{M}_{n}(F)=\{f \in C(F) \mid \forall \varepsilon>0$ найдется $n$-аналитическая мероморфная функция $h$ в $\mathbb{C}$, не имеющая особенностей на $F$ и удовлетворяющая условию $\left.\|f-h\|_{F}<\varepsilon\right\}$.

Таким образом, $\mathscr{E}_{n}(F)$ (соответственно, $\mathscr{M}_{n}(F)$ ) представляет собой класс функций из $C(F)$, каждая из которых равномерно на $F$ приближается $n$-аналитическими иельми функциями (соответственно, $n$-аналитическими мероморфными функциями в $\mathbb{C}$, не имеющими особенностей на $F)$.

Для компактного множества $X$ в $\mathbb{C}$ положим $\mathscr{P}_{n}(X)=\{f \in C(X) \mid \forall \varepsilon>0$ найдется $n$-аналитический полином $p$ такой, что $\left.\|f-p\|_{X}<\varepsilon\right\}$.

Для компактов $X$ и $Y$ в $\mathbb{C}$ с условием $X \subseteq Y$ через $\mathscr{R}_{n}^{Y}$ обозначим класс всех $n$-аналитических рациональных функций, не имеющих особенностей на $Y$, и определим

$$
\mathscr{R}_{n}^{Y}(X)=\left\{f \in C(X) \mid \forall \varepsilon>0 \exists r \in \mathscr{R}_{n}^{Y}:\|f-r\|_{X}<\varepsilon\right\}
$$

Отметим, что

$$
\mathscr{P}_{n}(X) \subseteq \mathscr{R}_{n}^{X}(X) \subseteq \mathscr{A}_{n}(X), \quad \mathscr{E}_{n}(F) \subseteq \mathscr{M}_{n}(F) \subseteq \mathscr{A}_{n}(F)
$$

и $\mathscr{E}_{n}(X)=\mathscr{P}_{n}(X)$.

В настоящей работе для случая $n \geqslant 2$ приводится ряд необходимых и достаточных условий на $X$ или $F$ (как мы увидим, эти условия не зависят от $n$ ), при которых возникают равенства в некоторых из только что упомянутых включений. В частности, для компактных множеств $X$ наши результаты сушественно усиливают основные результаты работы [1] (см. $§ 2)$.

Введем несколько определений и понятий, необходимых для формулировки наших результатов. Для произвольного собственного подмножества $S \subset \mathbb{C}$ обозначим через $S^{\circ}$ его внутренность, через $\partial S$ - границу, а через $\bar{S}$ - его замыкание. Риманову сферу $\mathbb{C} \cup\{\infty\}$ и открытый единичный круг $\{t \in \mathbb{C}|| t \mid<1\}$ обозначим через $\overline{\mathbb{C}}$ и $\Delta$ соответственно. Для компакта $X$ из $\mathbb{C}$ через $\widehat{X}$ обозначим топологическую (или полиномиальную) оболочку множества $X$, т. е. объединение $X$ со всеми ограниченными компонентами множества $\mathbb{C} \backslash X$ ( “дырами” этого $X$ ).

Пусть $f$ - ограниченная голоморфная функция на $\Delta$. По теореме Фату угловье граничные значения $f(t)$ существуют $|d t|$-почти всюду $(|d t|-$ п. в. ) на $\partial \Delta$. Следующее понятие было введено в [1].

ОПРЕДЕЛЕНИЕ 1. Ограниченная односвязная область $\Omega \subset \mathbb{C}$ называется неванлинновской, если найдутся конформное отображение $k: \Delta \rightarrow \Omega$ круга $\Delta$ на $\Omega$ и две ограниченные голоморфные функции $u, v$ в $\Omega$ (причем $v \not \equiv 0$ ) такие, что $|d t|$-п.в. на $\partial \Delta$ выполнено равенство

$$
\overline{k(t)}=\frac{u(k(t))}{v(k(t))}
$$


Как указано в [1], это определение корректно, т. е. не зависит от выбора $k$. Если $k_{1}, u, v$ удовлетворяют (2) и $k_{2}$ - какое-либо другое конформное отображение $\Delta$ на $\Omega$, то, записывая $k_{2}=k_{1} \circ k_{1}^{-1} \circ k_{2}$, мы видим, что $k_{2}, u, v$ также удовлетворяют (2). Более того, отношение $u / v$ (для неванлинновских областей) определено однозначно в $\Omega$. Действительно, если $k_{1}, u_{1}, v_{1}$ и $k_{2}, u_{2}, v_{2}$ удовлетворяют (2), то, как мы только что заметили, $k_{1}, u_{2}, v_{2}$ тоже удовлетворяют (2). По теореме Лузина-Привалова (см. $[2$, гл. $4, \S 2.5],[3$, гл. $3, \S$ D 3$])$ имеем

$$
\left(\frac{u_{1}}{v_{1}}\right) \circ k_{1}=\left(\frac{u_{2}}{v_{2}}\right) \circ k_{1}
$$

откуда $u_{1} / v_{1}=u_{2} / v_{2}$.

Класс всех неванлинновских областей будем обозначать через $\mathscr{N}$. Характеристика областей $\Omega$ класса $\mathcal{N}$ в терминах конформных отображений $\Delta$ на $\Omega$ приведена в $[1, \S 3]$; там же можно найти ряд содержательных примеров таких областей.

ОПРЕДЕЛЕНИЕ 2 . Область $\Omega \subset \mathbb{C}$ называется областью Каратеодори, если $\Omega$ ограничена и $\partial \Omega=\partial \Omega_{\infty}$, где $\Omega_{\infty}$ - неограниченная компонента множества $\mathbb{C} \backslash \bar{\Omega}$.

В примере 3 (см. $§ 4$ далее) приведена конструкция неванлинновских областей, не являющихся областями Каратеодори.

ОПРЕДЕЛЕНИЕ 3 . Пусть $A$-подмножество $\mathbb{C}$. Непустое подмножество $I \subset A$ назовем гранью множества $A$ (или $A$-гранью), если каждая точка $a \in I$ имеет окрестность $V$ в $\mathbb{C}$ такую, что найдется гомеоморфизм $h$ из $V \cap A$ на относительно открытое подмножество замкнутой верхней полуплоскости, удовлетворяюший условию $V \cap I=\{z \in V \mid \operatorname{Im} h(z)=0\}$ (таким образом, всякая грань локально является жордановой дугой); кроме того, мы всегда требуем, чтобыгрань $I$ являлась локально спрямляемой.

Заметим, что если $\Omega$ - открытое множество в $\mathbb{C}$, грань $I$ является $\bar{\Omega}$-гранью, a $f$ ограничена и голоморфна в $\Omega$, то угловые граничные значения $f(\zeta)$ сушествуют для почти всех $\zeta$ на $I$ относительно длины (на $I)[2$, гл. $3, \S 1.7]$.

Для произвольной ограниченной области $\Omega$ и относительно открытого подмножества $I$ на $\partial \Omega$ при $\varepsilon>0$ определим множества

$$
\begin{aligned}
& \Omega_{\varepsilon}(I)=\{z \in \Omega \mid \operatorname{dist}(z, \partial \Omega \backslash I)>\varepsilon\}, \\
& I_{\varepsilon}(\Omega)=\{z \in I \mid \operatorname{dist}(z, \partial \Omega \backslash I)>\varepsilon\} .
\end{aligned}
$$

При $I=\partial \Omega$ полагаем $\Omega_{\varepsilon}(I)=\Omega$ и $I_{\varepsilon}(\Omega)=\partial \Omega$.

ОПРЕДЕЛЕНИЕ 4 . Пусть $\Omega$ - ограниченная область в $\mathbb{C}$, и пусть $I-$ ее $\bar{\Omega}$-грань. Скажем, что $\Omega$ является $I$-неванлинновской (обозначим $\Omega \in \mathscr{N}(I)$ ), если найдется мероморфная в $\Omega$ функция $\varphi=\varphi_{\Omega}^{I}$, имеющая п. в. на $I$ угловые (из $\Omega$ ) граничные значения $\varphi(\zeta)$ с условиями $\varphi(\zeta)=\bar{\zeta}$ п.в. на $I$, причем для каждого достаточно малого $\varepsilon>0$ сушествуют ограниченные голоморфные в $\Omega_{\varepsilon}(I)$ функции $u_{\varepsilon}$ и $v_{\varepsilon}$ $\left(v_{\varepsilon} \not \equiv 0\right)$ такие, что $\varphi(\zeta)=u_{\varepsilon}(\zeta) / v_{\varepsilon}(\zeta)$ в $\Omega_{\varepsilon}(I)$.

Из упомянутой выше теоремы Лузина-Привалова следует, что если указанная в определении 4 функция $\varphi$ сушествует, то она единственна. Однако не ясно, всегда 
ли можно представить $\varphi$ как отношение ограниченных голоморфных функций во всем $\Omega$ (см. [4]).

Нетрудно видеть, что для жордановых областей $\Omega$ со спрямляемой границей $\partial \Omega$ имеет место соотношение

$$
\Omega \in \mathscr{N} \Longleftrightarrow \Omega \in \mathscr{N}(\partial \Omega)
$$

ОПРЕДЕЛЕНИЕ 5 . Пусть $\Omega$ - ограниченная область в $\mathbb{C}, \gamma_{1}$ и $\gamma_{2}-$ две жордановы (открытые) дуги, являющиеся $\bar{\Omega}$-гранями. Предположим, что при $\ell=1,2$ существуют окрестности $U_{\ell}$ множеств $\gamma_{\ell}$ в $\mathbb{C}$ такие, что $\Omega_{\ell}=\Omega \cap U_{\ell} \in \mathcal{N}\left(\gamma_{\ell}\right)$. Пусть $\varphi_{\ell}-$ мероморфная функция из определения 4 для пары $\left(\Omega_{\ell}, \gamma_{\ell}\right), \ell=1,2$. Назовем $\gamma_{1}$ и $\gamma_{2}$ неванлинновски $\Omega$-независимымми, если не сушествует пути в $\Omega$, вдоль которого два мероморфных элемента $\left(\Omega_{\ell}, \varphi_{\ell}\right), \ell=1,2$, были бы мероморфными продолжениями друг друга.

ЗАмечАниЕ 1. Из теоремы Лузина-Привалова следует, что если $\gamma_{1}$ и $\gamma_{2}$ неванлинновски $\Omega$-независимы, то $\Omega \notin \mathscr{N}(I)$ для всякой $\bar{\Omega}$-грани $I$, содержащей $\gamma_{1} \cup \gamma_{2}$. В частности, если $\gamma_{1}$ и $\gamma_{2}-$ аналитически независимые $\bar{\Omega}$-грани на $\partial \Omega$ (см. [1, $\S 3]$, [5]), то $\gamma_{1}$ и $\gamma_{2}$ также неванлинновски $\Omega$-независимы. Так, в каждом многоугольнике $\Omega$ любые две его стороны, не лежащие на одной прямой, неванлинновски $\Omega$-независимы.

Приведем еще два простых, но полезных примера.

Во-первых, пусть $\Omega_{0} \in \mathcal{N}$ и $I_{0}$ является $\bar{\Omega}_{0}$-гранью (например, $\Omega_{0}$ - открытьй круг, а $I_{0}$ - какое-либо непустое открытое подмножество его границы). Если $\Omega-$ произвольная подобласть в $\Omega_{0}$, а $I \subset I_{0}-\bar{\Omega}$-грань, то $\Omega \in \mathscr{N}(I)$.

Во-вторых, согласно [1, предложение 3.1$]$ образ $\Omega$ круга $\Delta$ при конформном отображении $z=\left((t+1)^{2}-4\right)^{2}$ является неванлинновской областью, для которой $\bar{\Omega}$-грани

$$
\gamma_{1}=\{z \in \partial \Omega \mid \operatorname{Re}(z)<0\} \cup\{0\}, \quad \gamma_{2}=\{z \in \partial \Omega \mid \operatorname{Re}(z)>0\}
$$

неванлинновски $\Omega$-зависимы (т. е. не независимы). При этом $\gamma_{1}$ и $\gamma_{2}$ даже не являются гладкими (а тем более, аналитическими) кривыми.

Замечание 1 и последние примеры показывают, что определения 4 и 5 существенно расширяют понятия неванлинновской области, функции Шварца аналитической кривой [5] и понятие аналитической независимости аналитических дуг.

\section{§ 2. Основные результаты}

Следуюшие две теоремы дают весьма полезные "редуктивные" условия для выполнения равенств $\mathscr{A}_{n}(F)=\mathscr{E}_{n}(F)$ и $\mathscr{A}_{n}(F)=\mathscr{M}_{n}(F)$ : они позволяют свести к соответствуюшим проблемам на компактных множествах проблемы аппроксимации функций на замкнуты $x$ множествах $n$-аналитическими целыми функциями (теорема 1 , которая устанавливает только необходимые условия) и $n$-аналитическими мероморфными функциями (теорема 2). Отметим, что (при $n=1)$ полные характеристики замкнутых множеств $F$, удовлетворяющих условию $\mathscr{A}_{1}(F)=\mathscr{E}_{1}(F)$ или $\mathscr{A}_{1}(F)=\mathscr{M}_{1}(F)$, получены Н.У. Аракеляном [6] и А.Х. Нерсесяном [7] соответственно. 
ТеОрема 1. Пусть $F$ - замкнутое подмножсество в $\mathbb{C}$ и $n \in \mathbb{N}$ фиксировано. Если $\mathscr{A}_{n}(F)=\mathscr{E}_{n}(F)$, то для всякой области Каратеодори $\Omega$ имеют место равенства

$$
\begin{aligned}
& \mathscr{A}_{n}(F \cap \bar{\Omega})=\mathscr{R}_{n}^{\bar{\Omega}}(F \cap \bar{\Omega}), \\
& \mathscr{A}_{n}(\overline{F \cap \Omega})=\mathscr{R}_{n}^{\bar{\Omega}}(\overline{F \cap \Omega}) .
\end{aligned}
$$

В частности, при дополнительном условии связности множества $\mathbb{C} \backslash \bar{\Omega}$ справедливы равенства

$$
\begin{aligned}
& \mathscr{A}_{n}(F \cap \bar{\Omega})=\mathscr{P}_{n}(F \cap \bar{\Omega}), \\
& \mathscr{A}_{n}(\overline{F \cap \Omega})=\mathscr{P}_{n}(\overline{F \cap \Omega}) .
\end{aligned}
$$

ПроБлемА 1. Верно ли, что при $n \geqslant 2$ (необходимые) условия $(3)$ (или $\left.\left(3^{\prime}\right)\right)$ являются также достаточными для выполнения равенства $\mathscr{A}_{n}(F)=\mathscr{E}_{n}(F) ?$

Tеорема 2. Пусть $F$ - замкнутое множество в $\mathbb{C}$, а $n \in \mathbb{N}$ фиксировано. Для выполнения равенства $\mathscr{A}_{n}(F)=\mathscr{M}_{n}(F)$ необходимо и достаточно, чтобь для любой области Каратеодори $\Omega$ выполнялось равенство

$$
\mathscr{A}_{n}(F \cap \bar{\Omega})=\mathscr{R}_{n}^{F \cap \bar{\Omega}}(F \cap \bar{\Omega}) .
$$

При $n=1$ из теоремы Аракеляна (см. [8, гл. IV, § 2C]) непосредственно вытекает, что условия $(3)\left(\right.$ и $\left.\left(3^{\prime}\right)\right)$ не являются достаточными для того, чтобы $\mathscr{A}_{1}(F)=$ $\mathscr{E}_{1}(F)$. Аналогичные условия не являются достаточными также в задаче аппроксимации целыми гармоническими функциями (см. [9, гл. $3, \S 10])$. Теорема 2 при $n=1$ приведена, например, в [8, гл. IV, § 1D]. Отметим, однако, что данная там формулировка должна быть исправлена: следует сказать “для любой области $K a-$ paтеодори $g "$, иначе утверждение не верно.

В $\S 4$ приведены примеры, иллюстрирующие теоремы 1 и 2 и показывающие, что в определенных ситуациях аппроксимация целыми $n$-аналитическими функциями (при $n \geqslant 2$ ) устроена проще, чем аппроксимация цельми голоморфными $(n=1)$ или целыми гармоническими функциями. Например, при $n \geqslant 2$ условие локальной связности множества $\overline{\mathbb{C}} \backslash F$ в точке $\infty$ уже не является необходимым (полезно сравнить последнее замечание с утверждениями теорем Аракеляна $[8$, гл. IV, $\S 2 \mathrm{C}]$ и Гардинера [9, следствие 3.21$])$.

Следующая теорема является локальной по характеру, хотя, безусловно, задача о совпадении пространств $\mathscr{A}_{n}(X)$ и $\mathscr{P}_{n}(X)$ не локальна в стандартном смысле (см. $[10$, гл. $2, \S 10])$. Эта теорема значительно усиливает результат $[1$, теоремa 2.2].

ТЕорема 3. Фиксируем произвольное $n \in \mathbb{N}$. Пусть $X-$ компактное множсество в $\mathbb{C}, a\left\{\Omega_{\ell}\right\}_{\ell \in L}-$ компоненты (связности) множества $(\widehat{X})^{\circ}$, не содержашиеся в $X$. Равенство

$$
\mathscr{A}_{n}(X)=\mathscr{P}_{n}(X)
$$

справедливо, если и только если равенства

$$
\mathscr{A}_{n}\left(X \cap \bar{\Omega}_{\ell}\right)=\mathscr{R}_{n}^{\bar{\Omega}_{\ell}}\left(X \cap \bar{\Omega}_{\ell}\right)
$$

верныл для всех $\ell \in L$. 
ЗАмЕчанИЕ 2 . Если $n=1$, то непосредственно из теоремы Мергеляна (см. $[8$, гл. III, $\S 2])$ получаем, что $(6) \Longleftrightarrow(X=\widehat{X}) \Longleftrightarrow(7)$, откуда вытекает справедливость теоремы 3 в этом случае.

Отметим также, что если $X$ является компактом Каратеодори (т.е. когда $\partial X=\partial \widehat{X})$, то $\left\{\Omega_{\ell}\right\}_{\ell \in L}-$ ограниченные компоненты множества $\mathbb{C} \backslash X$, так что с помощью теорем 3 и 1 получается теорема 2.2 из [1].

При использовании теоремы 3 важно уметь отвечать на следующий вопрос.

ПроБлЕма 2. Пусть $\Omega$ - область Каратеодори и $X$ - компактное подмножество в $\bar{\Omega}$ с условием $\mathscr{A}_{n}(X)=\mathscr{R}_{n}^{\bar{\Omega}}(X), n \geqslant 2$. При каких дополнительных условиях справедливо равенство $\mathscr{A}_{n}(X \cup \partial \Omega)=\mathscr{R}_{n}^{\bar{\Omega}}(X \cup \partial \Omega)$ ?

В работе [1] проблема 2 изучалась для случая, когда $\Omega$ - жорданова область, a $X$ строго содержится в $\Omega$ (см. $[1$, теорема 4.3$]$ ). Мы установим следуюший более сильньй результат.

ТЕОРема 4. Пусть $n \geqslant 2$ фиксировано, $\Omega$ - область Каратеодори, а Xкомпакт в $\bar{\Omega}$ с условием

$$
\mathscr{A}_{n}(X)=\mathscr{R}_{n}^{\bar{\Omega}}(X),
$$

причем множество $E:=\partial \Omega \backslash X$ является $\bar{\Omega}$-гранью. Пусть $\left\{D_{\ell}\right\}_{\ell \in L}-$ все компоненты множества $\Omega \backslash X$, для которых $E_{\ell}:=\partial D_{\ell} \backslash X \neq \varnothing$. Eсли $D_{\ell} \notin \mathscr{N}\left(E_{\ell}\right)$ для каждого $\ell \in L$, mо

$$
\mathscr{A}_{n}(X \cup \partial \Omega)=\mathscr{R}_{n}^{\bar{\Omega}}(X \cup \partial \Omega) .
$$

СлЕДСТВИЕ 1. Фиксируем $n \geqslant 2$. Пусть $\Omega$-область Каратеодори, $\mathbb{C} \backslash \bar{\Omega}$ связно и $\partial \Omega$ содержит две неванлинновски $\Omega$-независимые дуги $\gamma_{1}$ и $\gamma_{2}$. Пусть $X-$ компакт в $\Omega$ с условием $\mathscr{A}_{n}(X)=\mathscr{P}_{n}(X)$. Тогда $\mathscr{A}_{n}(K)=\mathscr{P}_{n}(K)$, əде $K=X \cup \partial \Omega$.

Отметим, что следствие 1 и, в частности, теорема 4 не верны при $n=1$.

\section{§ 3. Доказательства}

ДоКАЗАТЕЛЬСТво ТЕОРЕмЫ 1 . МЫ установим только условие (3), доказательство $\left(3^{\prime}\right)$ аналогично. Более того, по теореме Рунге равенства (4) и $\left(4^{\prime}\right)$ вытекают из $(3)$ и $\left(3^{\prime}\right)$ соответственно.

Предположим, что $n \in \mathbb{N}$ фиксировано, $\mathscr{A}_{n}(F)=\mathscr{E}_{n}(F), \Omega$ - область Каратеодори, $f \in \mathscr{A}_{n}(F \cap \bar{\Omega})$. Сначала по теореме Брауэра продолжим $f$ до функции, снова обозначаемой через $f$, которая непрерьвна на $\mathbb{C}$ и имеет компактньй носитель. Теперь применим аппроксимационную схему Витушкина [11], надлежащим образом адаптированную к рассматриваемой ситуации. Кратко опишем этот метод. Детали можно найти в $[1$, доказательство предложения 2.5$]$.

Положим $\mathscr{L}=\bar{\partial}^{n}$, и пусть $\Phi(z)=\bar{z}^{n-1}((n-1) ! \pi z)^{-1}$ - фундаментальное решение для $\mathscr{L}$. Пусть $\partial:=\partial / \partial z$, а $B(a, r)$ - открытый круг радиуса $r>0$ с центром в точке $a$. Фиксируем произвольное $\delta \in(0,1)$ и построим $\delta$-разбиение 
единицы следуюшим образом. Для каждого $j=\left(j_{1}, j_{2}\right) \in \mathbb{Z}^{2}$ положим $a_{j}=$ $j_{1} \delta+i j_{2} \delta, B_{j}=B\left(a_{j}, \delta\right)$ и выберем $\varphi_{j} \in C_{0}^{\infty}\left(B\left(a_{j}, \delta\right)\right)$ так, что $0 \leqslant \varphi_{j} \leqslant 1$, $\sum_{j \in \mathbb{Z}^{2}} \varphi_{j} \equiv 1$ и $\left\|\bar{\partial}^{r} \partial^{s} \varphi_{j}\right\| \leqslant A \delta^{-(r+s)}, 1 \leqslant r+s \leqslant n$, где $A$ зависит только от $n$. Определим $f_{j}=\Phi *\left(\varphi_{j} \mathscr{L} f\right)$. Ясно, что $f_{j} \in \mathscr{A}_{n}(F)$. Положим

$$
J=\left\{j \in \mathbb{Z}^{2} \mid B\left(a_{j}, \delta\right) \cap \operatorname{supp} f \neq \varnothing\right\}
$$

и отметим, что множество $J$ конечно. Имеем $\mathscr{L} f_{j}=\varphi_{j} \mathscr{L} f$ (в обобщенном смысле) и $f=\sum_{j \in J} f_{j}$. Пусть

$J_{1}=\left\{j \in J \mid B_{j} \subset \Omega\right\}, \quad J_{2}=\left\{j \in J \mid B_{j} \cap \bar{\Omega}=\varnothing\right\}, \quad J_{3}=\left\{j \in J \mid B_{j} \cap \partial \Omega \neq \varnothing\right\}$.

Ясно, что $J=J_{1} \cup J_{2} \cup J_{3}$.

Если $j \in J_{1}$, то $f_{j} \in \mathscr{A}_{n}(F)=\mathscr{E}_{n}(F) \subset \mathscr{R}_{n}^{\bar{\Omega}}(F \cap \bar{\Omega})$. При $j \in J_{2}$ имеем $f_{j} \in \mathscr{R}_{n}^{\bar{\Omega}}(F \cap \bar{\Omega})$ по теореме Рунге.

Остается рассмотреть случай $j \in J_{3}$. Пусть $\delta<\operatorname{diam}(\Omega) / 2$. Поскольку $\Omega-$ область Каратеодори, то для каждого $j \in J_{3}$ можно найти $a_{j}^{*} \in B\left(a_{j}, \delta\right) \backslash \bar{\Omega}$ и жорданову кривую $\gamma_{j}$ в $\overline{B\left(a_{j}^{*}, \delta\right)} \backslash \bar{\Omega}$ с начальной точкой $a_{j}^{*}$ и конечной точкой на $\partial B\left(a_{j}^{*}, \delta\right)$. Но тогда можно построить (см. $[1$, доказательство предложения 2.5]) функции $g_{j}, n$-аналитические вне $\gamma_{j}$ и, следовательно, принадлежашие $\mathscr{R}_{n}^{\bar{\Omega}}(F \cap \bar{\Omega})$, такие, что

$$
\left\|\sum_{j \in J_{3}}\left(f_{j}-g_{j}\right)\right\|_{F \cap \bar{\Omega}} \longrightarrow 0, \quad \delta \rightarrow 0 .
$$

Нужная аппроксиманта имеет вид $g=\sum_{j \in J_{1} \cup J_{2}} f_{j}+\sum_{j \in J_{3}} g_{j}$. Теорема доказана.

ДОКАЗАТЕЛЬСТВО ТЕОРЕМЫ 2. В предПоЛожениИ, что $\mathscr{A}_{n}(F)=\mathscr{M}_{n}(F)$ и $f \in \mathscr{A}_{n}(F \cap \bar{\Omega})$, доказательство необходимости практически дословно повторяет доказательство теоремы 1. Утверждение достаточности новым результатом не является, оно может быть получено аналогично $[12, \S 1$ и $\S 2]$ (см. также [12, теорема 3]).

ДоКАЗАТЕЛЬСТво ТЕОРЕМЫ 3 . Заметим, что все $\Omega_{\ell}, \ell \in L$, являются областями Каратеодори, так что по теореме 1 из равенства (6) вытекает (7).

Для доказательства достаточности условий (7) рассмотрим произвольную конечную комплексную борелевскую меру $\mu$ с носителем на $X$ и условием $\mu \perp$ $\mathscr{P}_{n}(X)$. Установим, что $\mu \perp \mathscr{A}_{n}(X)$ при условии выполнения (7).

Сначала отметим, что если множество индексов $L$ пусто, т. е. $X$ имеет связное дополнение, то условие (7) отсутствует и равенство (6) следует из [13, теорема 2] и теоремы Рунге. Теперь предположим, что $L \neq \varnothing$, и фиксируем $\ell \in L$. Согласно $[1$, лемма 2.4] найдется последовательность полиномов комплексного переменного $\left\{q_{j}\right\}_{j=1}^{\infty}$ со следуюшими свойствами: $\left\|q_{j}\right\|_{\hat{X}} \leqslant C$ ( $C$-некоторая абсолютная константа), $q_{j} \rightarrow 1$ равномерно на компактах в $\Omega_{\ell}, q_{j} \rightarrow 0$ равномерно на компактах в $(\widehat{X})^{0} \backslash \Omega_{\ell}$ при $j \rightarrow \infty$. Пусть $\mathscr{F}$ - некоторое счетное всюду плотное семейство в $C(X)$. Применяя диагональный процесс для выделения нужной подпоследовательности, можно дополнительно считать, что последовательность $\int f q_{j} d \mu$ сходится при всех $f \in \mathscr{F}$. Из свойства плотности $\mathscr{F}$ в $C(X)$ вытекает, 
что последовательность $\int f q_{j} d \mu$ сходится также и при всех $f \in C(X)$. Последнее условие определяет непрерывный линейный функционал в $C(X)$. Пусть $\mu_{\Omega_{\ell}}-$ мера, представляющая этот функционал. По определению $q_{j} \mu \rightarrow \mu_{\Omega_{\ell}}$ в $*$-слабой топологии при $j \rightarrow \infty$. Следовательно, supp $\mu_{\Omega_{\ell}} \subset\left(X \cap \bar{\Omega}_{\ell}\right) \cup \partial \widehat{X}$ и $\mu_{\Omega_{\ell}} \perp \mathscr{P}_{n}(X)$.

Напомним, что

$$
\hat{\nu}(\zeta)=\frac{1}{2 \pi i} \int \frac{d \nu(z)}{z-\zeta}
$$

есть преобразование Коши (конечной) меры $\nu$. Мы утверждаем, что

$$
\widehat{\bar{z}^{s} \mu}\left(z_{0}\right)=0 \quad \forall z_{0} \in \Omega_{\ell} \backslash X, \quad s=0, \ldots, n-1 .
$$

Действительно, поскольку $\widehat{\bar{z}^{s} \mu}\left(z_{0}\right)=\widehat{\overline{z^{s}} \mu_{\Omega_{\ell}}}\left(z_{0}\right)$ при $z_{0} \in \Omega_{\ell} \backslash X$ (см. $[1$, доказательство теоремы $2.2,(2)]$ ), можно (без потери обшности) с самого начала считать, что

$$
\operatorname{supp} \mu \subset\left(X \cap \bar{\Omega}_{\ell}\right) \cup \partial \widehat{X}
$$

Однако если (11) выполнено для меры $\mu$, то справедливо включение supp $\mu_{\Omega_{\ell}} \subset$ $X \cap \bar{\Omega}_{\ell}$. В самом деле, так как $q_{j} \rightarrow 0$ на $(\widehat{X})^{0} \backslash \Omega_{\ell}$, то

$$
\widehat{\bar{z}^{s} \mu_{\Omega_{\ell}}}(w)=0 \quad \forall w \in\left[(\widehat{X})^{0} \backslash \Omega_{\ell}\right] \cup[\mathbb{C} \backslash \widehat{X}], \quad s=0, \ldots, n-1
$$

Поскольку $\mu$ не имеет атомов на $\partial \widehat{X}$ (см. [14, доказательство леммы 9]), функция

$$
f_{\Omega_{\ell}}(w)=\frac{1}{\pi} \int \frac{\bar{z}-\bar{w}}{z-w} d \mu_{\Omega_{\ell}}(z)
$$

непрерывна на $\mathbb{C} \backslash \bar{\Omega}_{\ell}$, и ввиду условий $(12)$ она равна нулю вне $\bar{\Omega}_{\ell}$. Отсюда вытекает, что $\left.\mu_{\Omega_{\ell}}\right|_{\mathbb{C} \backslash \bar{\Omega}_{\ell}}=0$, так как $\bar{\partial}^{2} f_{\Omega_{\ell}}=\mu_{\Omega_{\ell}}$ в обобшенном смысле. Таким образом,

$$
\mu_{\Omega_{\ell}} \perp \mathscr{R}_{n}^{\bar{\Omega}_{\ell}}\left(X \cap \bar{\Omega}_{\ell}\right)=\mathscr{A}_{n}\left(X \cap \bar{\Omega}_{\ell}\right)
$$

и соотношение (10) имеет место.

Теперь, поскольку $\ell$ было произвольным в условии (10), имеем

$$
\widehat{\bar{z}^{s} \mu}\left(z_{0}\right)=0 \quad \forall z_{0} \in \mathbb{C} \backslash X, \quad s=0, \ldots, n-1
$$

так что $\mu \perp \mathscr{R}_{n}^{X}(X)$.

В завершение доказательства остается показать, что $\mathscr{R}_{n}^{X}(X)=\mathscr{A}_{n}(X)$. Для этой цели снова применим технику Витушкина, используя обозначения из приведенного выше доказательства теоремы 1.

Возьмем произвольную $f \in \mathscr{A}_{n}(X)$ и продолжим ее до функции (снова обозначаемой через $f$ ), непрерьвной на всем $\mathbb{C}$ и имеющей компактный носитель. Пусть $\left\{f_{j}\right\}$ - локализованные функции, соответствующие разбиению единищы $\left\{B_{j}, \varphi_{j}\right\}$. Если $B_{j} \cap \widehat{X}=\varnothing$ или $B_{j} \cap \partial \widehat{X} \neq \varnothing$, то соответствуюшие функции $f_{j}$ в приближении не нуждаются (см. доказательство теоремы 1 ). Остается рассмотреть случай, когда $B_{j} \subset(\widehat{X})^{\circ}$. В этой ситуации $B_{j}$ лежит в некоторой компоненте (связности) $\Omega$ 
множества $(\widehat{X})^{\circ}$. Отметим, что если $\Omega \subset X^{\circ}$, то $f_{j} \equiv 0$. Если же $\Omega \not \subset X^{\circ}$, то $\Omega=\Omega_{\ell}$ для некоторого $\ell \in L$. Поскольку

$$
f \in \mathscr{A}_{n}\left(X \cap \bar{\Omega}_{\ell}\right)=\mathscr{R}_{n}^{\bar{\Omega}_{\ell}}\left(X \cap \bar{\Omega}_{\ell}\right),
$$

то можно найти последовательность $\left\{g_{m}\right\}_{m=1}^{\infty}$, где каждая $g_{m}$ является $n$-аналитической в некоторой окрестности $U_{m}$ множества $X \cap \bar{\Omega}_{\ell}$, такую, что после надлежащего продолжения функции $g_{m}$ до непрерьвной функции (которая снова обозначается через $\left.g_{m}\right)$ на всем $\bar{\Omega}_{\ell}$ имеет место $\left\|f-g_{m}\right\|_{\bar{\Omega}_{\ell}} \rightarrow 0$ при $m \rightarrow \infty$. Используя хорошо известные свойства оператора Витушкина, получаем

$$
\left\|\Phi *\left(\varphi_{j} \mathscr{L} g_{m}\right)-f_{j}\right\|_{X}=\left\|\Phi *\left(\varphi_{j}\left(\mathscr{L} g_{m}-\mathscr{L} f\right)\right)\right\|_{X} \leqslant C\left\|g_{m}-f\right\|_{\bar{B}_{j}} \rightarrow 0
$$

при $m \rightarrow \infty$. Ясно, что $\Phi *\left(\varphi_{j} \mathscr{L} g_{m}\right) \in \mathscr{R}_{n}^{X}(X)$. Доказательство теоремы завершено.

ДоКАЗАТЕЛЬСТВо ТЕОРЕМЫ 4. Будем доказывать от противного. Пусть $K=$ $X \cup \partial \Omega$, и предположим, что

$$
\mathscr{A}_{n}(K) \neq \mathscr{R}_{n}^{\bar{\Omega}}(K) .
$$

Покажем, что обязательно найдется $\ell \in L$ такое, что $D_{\ell} \in \mathscr{N}\left(E_{\ell}\right)$.

Из (13) следует, что найдется мера $\sigma$ с носителем на $K$ такая, что $\sigma \perp \mathscr{R}_{n}^{\bar{\Omega}}(K)$, но $\sigma \not \perp \mathscr{A}_{n}(K)$. Заметим, что мера $\left.\sigma\right|_{E}$ не является нулевой, так как в противном случае мы бы имели $\operatorname{supp} \sigma \subset X$, что противоречит (8). Поскольку $E=\bigcup_{\ell \in L} E_{\ell}$ и $L$ не более чем счетно, можно выбрать $\ell$ такое, что $\left.\sigma\right|_{E_{\ell}}$ также не является нулевой. Фиксируем это $\ell$. Докажем, что $D_{\ell} \in \mathscr{N}\left(E_{\ell}\right)$. Пусть $X_{\ell}=\bar{\Omega} \backslash\left(D_{\ell} \cup E_{\ell}\right)$, так что $\partial X_{\ell} \subset \partial \Omega \cup \partial D_{\ell}$. Фиксируем $\varepsilon>0$ настолько малым, чтобы $E_{\ell}^{\varepsilon}:=\left\{z \in E_{\ell} \mid \operatorname{dist}\left(z, X_{\ell}\right) \geqslant \varepsilon\right\} \neq \varnothing$ и $\left.\sigma\right|_{E_{\ell}^{\varepsilon}} \neq 0$. По определению $E_{\ell}$

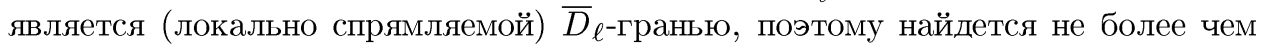
счетное семейство попарно не пересекаюшихся (открытых) жордановых дуг $\left\{\gamma_{j}\right\}_{j \in J_{\ell}}$ такое, что $E_{\ell}=\bigcup_{j \in J_{\ell}} \gamma_{j}$. Более того, так как $E_{\ell}^{\varepsilon}-$ компакт в $\partial D_{\ell}$, а каждая $\gamma_{j}$ открыта в $\partial D_{\ell}$, найдется конечное подмножество $J \subset J_{\ell}$ с условием $E_{\ell}^{\varepsilon} \subset \bigcup_{j \in J} \gamma_{j}$.

Для каждого $j \in J$ выберем точки $a_{j}$ и $b_{j}$ на $\gamma_{j}$ со следующими условиями: $\gamma_{j}$ имеет касательные в $a_{j}$ и $b_{j}$. Путь $\widetilde{\gamma}_{j}$, представляющий собой часть пути $\gamma_{j}$ строго между $a_{j}$ и $b_{j}$ и направленный от $a_{j}$ к $b_{j}$, имеет положительную ориентацию по отношению к $\Omega$; кроме того, $E_{\ell}^{\varepsilon} \subset \bigcup_{j \in J} \widetilde{\gamma}_{j}=: \Gamma_{1}$ и $\left.\sigma\right|_{\Gamma_{1}} \neq 0$. Заметим, что

$$
\operatorname{dist}\left(z, X_{\ell}\right)<\varepsilon, \quad z \in E_{\ell} \backslash \Gamma_{1} \text {. }
$$

Пусть $\Omega_{\infty}$ - неограниченная компонента (связности) множества $\overline{\mathbb{C}} \backslash \bar{\Omega}$. Тогда $\Omega_{\infty}$ односвязна, так как $\partial \Omega_{\infty}=\partial \Omega$ связна, причем каждая $\widetilde{\gamma}_{j}$ является $\bar{\Omega}_{\infty}$-гранью (здесь мы естественньм образом расширяем определение 3 на области в $\overline{\mathbb{C}}$, содержашие точку $\infty)$. Будем считать, что $J=\{1,2, \ldots, N\}$ и $\widetilde{\gamma}_{j}$ идут в последовательном порядке на $\partial \Omega_{\infty}$. Отметим, что $\widetilde{\gamma}_{j}$ имеют отрицательную ориентацию относительно $\Omega_{\infty}$. Следовательно, для каждого $j \in J$ можно найти жордановы спрямляемые дуги $\beta_{j}$ в $\Omega_{\infty}$, начинаюшиеся в $b_{j}$ и заканчиваюшиеся в $a_{j+1}$, ортогональные к $\gamma_{j}$ в $b_{j}$ и ортогональные к $\gamma_{j+1}$ в $a_{j+1}\left(\right.$ где $a_{N+1}:=a_{1}$ и $\left.\gamma_{N+1}=\gamma_{1}\right)$. 
Кроме того, можно дополнительно требовать, чтобы кривая $\Gamma=\bigcup_{j \in J}\left(\widetilde{\gamma}_{j} \cup \beta_{j}\right)$ была спрямляемой жордановой кривой, которая окружает и пересекает некасательно компакт

$$
Y=X_{\ell} \cup\left(E_{\ell} \backslash\left(\bigcup_{j \in J} \widetilde{\gamma}_{j}\right)\right),
$$

а также имеет положительную ориентацию относительно области $G$, ограниченной Г. Отметим, что $Y \subset G \cup\left(\bigcup_{j \in J}\left\{a_{j}, b_{j}\right\}\right), \operatorname{supp} \sigma \subset Y \cup \Gamma_{1}, \Gamma_{1} \subset \Gamma$, и напомним, что $\left.\sigma\right|_{\Gamma_{1}} \neq 0$. Более того, так как $\Gamma$ пересекает $Y$ некасательно, то при любом $z \in Y$ имеем

$$
\operatorname{dist}(z, \Gamma) \asymp \operatorname{dist}\left(z, \bigcup_{j \in J}\left\{a_{j}, b_{j}\right\}\right) .
$$

Положим $\sigma_{0}=\left(\prod_{j \in J}\left(z-a_{j}\right)\left(z-b_{j}\right)\right) \sigma$. Имеем $\sigma_{0} \perp \mathscr{P}_{n}(Y \cup \Gamma)$, поскольку последнее свойство справедливо для $\sigma$. Далее необходима следующая лемма.

ЛЕмма 1. Пусть $\mu$ - мера с условием, что $\left(\prod_{j \in J}\left|z-a_{j}\right|\left|z-b_{j}\right|\right)^{-1} \mu-$ конечная мера на $Y$. Тогда мера $\left.\hat{\mu}(\zeta) d \zeta\right|_{\Gamma}$ конечна и

$$
\left(\mu+\left.\hat{\mu}(\zeta) d \zeta\right|_{\Gamma}\right) \perp \mathscr{P}_{1}(Y \cup \Gamma)
$$

ДокАЗАТЕЛЬСтво. Докажем сначала конечность меры $\left.\hat{\mu}(\zeta) d \zeta\right|_{\Gamma}$ :

$$
\begin{aligned}
\int_{\Gamma}|\hat{\mu}(\zeta)||d \zeta| & =\frac{1}{2 \pi} \int_{\Gamma}\left|\int_{Y} \frac{d \mu(z)}{z-\zeta}\right| d \zeta\left|\leqslant \int_{Y}\left(\int_{\Gamma} \frac{|d \zeta|}{|z-\zeta|}\right)\right| d \mu(z) \mid \\
& \leqslant c \int_{Y} \frac{|\Gamma|}{\prod_{j \in J}\left|z-a_{j}\right|\left|z-b_{j}\right|}|d \mu(z)|<\infty,
\end{aligned}
$$

где $c>0$ - постоянная, $|\Gamma|$ - длина контура Г и предпоследнее неравенство вытекает из (14).

Последняя оценка позволяет воспользоваться теоремой Фубини для доказательства (15). Действительно, если $p$ - полином комплексного переменного, то

$$
\begin{aligned}
\int_{Y} p(z) d \mu(z)+\int_{\Gamma} p(\zeta) \hat{\mu}(z) d \zeta & =\int_{Y} p(z) d \mu(z)+\int_{\Gamma} p(\zeta)\left(\frac{1}{2 \pi i} \int_{Y} \frac{d \mu(z)}{z-\zeta}\right) d \zeta \\
& =\int_{Y} p(z) d \mu(z)+\int_{Y}\left(\frac{1}{2 \pi i} \int_{\Gamma} \frac{p(\zeta)}{z-\zeta} d \zeta\right) d \mu(z)=0
\end{aligned}
$$

по формуле Коши. Лемма доказана.

Теперь применим лемму 1 к мерам $\mu_{s}:=\left.\bar{z}^{s} \sigma_{0}\right|_{Y}, s=0, \ldots, n-1$. Имеем

$$
\left(\bar{z}^{s} \sigma_{0}-\left(\mu_{s}+\left.\hat{\mu}_{s}(\zeta) d \zeta\right|_{\Gamma}\right)\right) \perp \mathscr{P}_{1}(\Gamma)
$$

откуда по теореме Привалова $[2$, гл. $3, \S 7]$ (аналог известной теоремы Риссов для жордановых областей со спрямляемой гранищей) для каждого $s \in\{0, \ldots, n-1\}$ найдется функция $h_{s}$ из класса Смирнова $E_{1}(G)$ такая, что

$$
\bar{z}^{s} \sigma_{0}=\mu_{s}+\left.\hat{\mu}_{s}(\zeta) d \zeta\right|_{\Gamma}+\left.h_{s}(\zeta) d \zeta\right|_{\Gamma}
$$


Полагая $s=0$ в (16), умножая полученное уравнение на $\bar{z}^{s}, s=0, \ldots, n-1$, и учитывая, что $\mu_{s}=\bar{z}^{s} \mu_{0}$, имеем

$$
\bar{z}^{s} \sigma_{0}=\bar{z}^{s} \mu_{0}+\left.\bar{\zeta}^{s} \hat{\mu}_{0}(\zeta) d \zeta\right|_{\Gamma}+\left.\bar{\zeta}^{s} h_{0}(\zeta) d \zeta\right|_{\Gamma} .
$$

Вычитая последнее равенство из (16), при $s=0, \ldots, n-1$ получаем

$$
\left.\bar{\zeta}^{s}\left(\hat{\mu}_{0}(\zeta)+h_{0}(\zeta)\right) d \zeta\right|_{\Gamma}=\left.\left(\hat{\mu}_{s}(\zeta)+h_{s}(\zeta)\right) d \zeta\right|_{\Gamma} .
$$

Поскольку $\left.\sigma_{0}\right|_{\Gamma_{1}}=\left.\left(\hat{\mu}_{0}(\zeta)+h_{0}(\zeta)\right) d \zeta\right|_{\Gamma_{1}} \neq 0$, то по теореме Лузина-Привалова получаем, что $\hat{\mu}_{0}(z)+h_{0}(z) \not \equiv 0$ в $D_{\ell}$, так что угловые граничные значения $\hat{\mu}_{0}(\zeta)+$ $h_{0}(\zeta)$ отличны от нуля для почти всех $\zeta \in \Gamma_{1}$, откуда

$$
\bar{\zeta}^{s}=\frac{\hat{\mu}_{s}(\zeta)+h_{s}(\zeta)}{\hat{\mu}_{0}(\zeta)+h_{0}(\zeta)}
$$

п. в. на $\Gamma_{1}$. Так как каждая функция $\hat{\mu}_{s}$ ограничена вне $\varepsilon$-окрестности множества $Y$, а каждая $h_{s}$ есть отношение двух ограниченных голоморфных функций в $G$, то, используя простые вычисления, получаем (полагая $s=1$ ), что $\bar{\zeta}=u_{\varepsilon}(\zeta) / v_{\varepsilon}(\zeta)$ п.в. на $\Gamma_{1}$ для некоторых функций $u_{\varepsilon}$ и $v_{\varepsilon}$, ограниченных и голоморфных на $\{z \in$ $\left.D_{\ell} \mid \operatorname{dist}(z, K)>\varepsilon\right\}$. Последнее означает, что $D_{\ell} \in \mathscr{N}\left(E_{\ell}\right)$. Теорема доказана.

ДОКАЗАТЕЛЬСТВО СЛЕДСТВИЯ 1. Из условия ясно, что $\gamma_{1} \cap \gamma_{2}=\varnothing$. Пусть

$$
X_{1}=X \cup\left(\partial \Omega \backslash\left(\gamma_{1} \cup \gamma_{2}\right)\right) .
$$

Следствие вытекает непосредственно из теоремы 4 , примененной к $X_{1}$. Таким образом, остается проверить, что условия теоремы 4 выполнены в указанном случае.

Поскольку $\mathscr{A}_{n}(X)=\mathscr{P}_{n}(X), \widehat{X}$ содержится в $\Omega$ и $\partial \Omega \backslash\left(\gamma_{1} \cup \gamma_{2}\right)$ имеет связное дополнение, то по теореме Рунге имеем

$$
\mathscr{A}_{n}\left(X_{1}\right)=\mathscr{P}_{n}\left(X_{1}\right) .
$$

Кроме того, в указанном случае $E:=\partial \Omega \backslash X_{1}=\gamma_{1} \cup \gamma_{2}$ и совокупность $\left\{D_{\ell}\right\}_{\ell \in L}$ состоит всего из одной области $D_{1}=\Omega \backslash \widehat{X}$. Из замечания 1 следует, что $D_{1} \notin$ $\mathscr{N}\left(\gamma_{1} \cup \gamma_{2}\right)$. Таким образом, теорема 4 применима и следствие доказано.

\section{§ 4. Примеры}

ПримеР 1. Сначала приведем пример, показывающий, что теорема 1 перестает быть верной, если мы заменим $\mathscr{R}_{n}^{\bar{\Omega}}(F \cap \bar{\Omega})$ или $\mathscr{R}_{n}^{\bar{\Omega}}(\overline{F \cap \Omega})$ на $\mathscr{P}_{n}(F \cap \bar{\Omega})$ или $\mathscr{P}_{n}(\overline{F \cap \Omega})$ в $(3)$ или $\left(3^{\prime}\right)$ соответственно.

Пусть $U_{1}$ - открытая ограниченная "лента", которая накручивается на окружность $\{|z|=1\}$ и аккумулируется к ней извне. Говорят, что $U_{1}$ - "рог изобилия" (см. $[10$, гл. VI, $\S 5$, рис. 4$]$ или $[1, \S 3])$. Заметим, что $U_{1}$ является областью Каратеодори с тем условием, что $\mathbb{C} \backslash \bar{U}_{1}$ не связно.

Пусть $F=\{|z| \leqslant 1\} \cup \bar{U}_{1}$ и $\Omega=U_{1}$. Отметим, что $F$ - компакт Каратеодори, т. е. $\partial F=\partial \widehat{F}$, и что $F \cap \bar{\Omega}=\overline{F \cap \Omega}=\bar{\Omega}$. Для всех $n \in \mathbb{N}$ имеет место $\mathscr{A}_{n}(F)=$ $\mathscr{P}_{n}(F)$ согласно [13, теорема 2], а также $\mathscr{A}_{n}(F \cap \bar{\Omega})=\mathscr{R}_{n}^{\bar{\Omega}}(F \cap \bar{\Omega})$ согласно $[1$, предложение 2.5$]$, однако $\mathscr{A}_{n}(F \cap \bar{\Omega}) \neq \mathscr{P}_{n}(F \cap \bar{\Omega})$ ввиду [1, теорема 2.2, (2)]. 
ПРИмЕР 2. Следующий пример применения теоремы 3 не может быть получен с помошью $[1$, теорема $2.2,(2)]$.

Рассмотрим опять "рог изобилия". Однако теперь открытая “лента" $U_{2}$ наматывается снаружи эллипса $\left\{x^{2}+2 y^{2}=1\right\}$ и аккумулируется к этому эллипсу. Пусть $X$ - объединение $\bar{U}_{2}$ и сегмента $[-a, a]$, где $a>0$ и указанньй сегмент не содержит фокусы эллипса. Пусть $\Omega=\left\{x^{2}+2 y^{2}<1\right\}$ - внутренняя область рассматриваемого эллипса. Отметим, что $\Omega \notin \mathscr{N}$ (см. $[1, \S 3]), \Omega$ - единственная компонента множества $(\widehat{X})^{\circ}$, которая не содержится в $X$, и $X$ не является компактом Каратеодори (т. е. теорема $2.2,(2)$ из [1] не применима в этой ситуации), причем $X \cap \bar{\Omega}=\partial \Omega \cup[-a, a]$. Фиксируем $n \in \mathbb{N}, n \geqslant 2$. Из [1, теорема 4.3, (2)] следует, что

$$
\mathscr{A}_{n}(X \cap \bar{\Omega})=\mathscr{P}_{n}(X \cap \bar{\Omega})=\mathscr{R}_{n}^{\bar{\Omega}}(X \cap \bar{\Omega}),
$$

откуда по теореме 3 получаем $\mathscr{A}_{n}(X)=\mathscr{P}_{n}(X)$.

ПримеР 3. Приведем примеры неванлинновских областей, не являюшихся областями Каратеодори, и обсудим важный в приложениях результат $[1$, теорема $2.2,(1)]$.

Пусть $\Omega_{1}$ - образ круга $\Delta$ при конформном отображении $z=k_{1}(t)=(t+\sqrt{2})^{4}$. Очевидно, что $\Omega_{1}$ не является областью Каратеодори. С другой стороны, $\Omega_{1}$ является неванлинновской областью согласно [1, предложение 3.1$]$ (в обозначениях последнего утверждения достаточно взять $u_{1}(t)=k_{1}(t) / t^{4}$ и $\left.v_{1}(t)=1 / t^{4},|t|>1\right)$. Аналогично, чтобы получить пример неванлинновской области с $m$ ограниченными компонентами дополнения ( $m \in \mathbb{N}, m>1)$, достаточно рассмотреть образ $\Omega_{m}$ круга $\Delta$ при конформном отображении

$$
z=k_{m}(t)=\left(a_{m}+k_{m-1}(t)\right)^{2}
$$

где положительные константы $a_{m}$ подбираются по индукции надлежащим образом.

В $[1$, теорема $2.2,(1)]$ доказано, что для областей Каратеодори $\Omega$ при $n>1$ вьполнено

$$
C(\partial \Omega) \neq \mathscr{R}_{n}^{\bar{\Omega}}(\partial \Omega) \Longleftrightarrow \Omega \in \mathscr{N} .
$$

Возникает естественный вопрос: насколько важно требование, чтобы область $\Omega$ была каратеодориевской? Из доказательства теоремы $2.2,(1)$ работы [1] видно, что в части $\Leftarrow$ формулы $(17)$ это требование излишне. Приведенный только что пример показывает, что данное замечание существенно. С другой стороны, пример $\Omega=\Delta \backslash[0,1)$ (неневанлинновской и некаратеодориевской области) показывает, что $C(\partial \Omega) \neq \mathscr{R}_{n}^{\bar{\Omega}}(\partial \Omega)$, так что указанная теорема без условия каратеодориевости в части $\Rightarrow$ формулы (17) не верна.

ПримеР 4 . Приведем пример нигде не плотного замкнутого множества $F$ в $\mathbb{C}$ такого, что $\overline{\mathbb{C}} \backslash F$ связно, не является локально связным в $\infty$, однако, тем не менее, $F$ есть множество карлемановской аппроксимации (см. $[8$, гл. IV,$\S 3])$ посредством $n$-аналитических функций при (любом фиксированном) $n \geqslant 2$. В частности, $\mathscr{A}_{n}(F)=C(F)=\mathscr{E}_{n}(F)$. Это утверждение по существу противоположно соответствуюшему утверждению (случай $n=1$ ) теоремы Аракеляна $[8$, гл. IV, $\S 2]$, 
а также результатам о гармонических карлемановских аппроксимациях $[9$, теорема 4.6].

При $k=2,3,4, \ldots$ положим

$$
\begin{aligned}
& A_{k}=\left\{x=\frac{1}{k}, 0 \leqslant y \leqslant k\right\}, \\
& B_{k}=\left\{x=\frac{1}{2}\left(\frac{1}{k}+\frac{1}{k-1}\right), 0 \leqslant y \leqslant k\right\}, \\
& C_{k}=\left\{\frac{1}{k} \leqslant x \leqslant \frac{1}{2}\left(\frac{1}{k}+\frac{1}{k-1}\right), y=k\right\},
\end{aligned}
$$

и пусть

$$
F=\left(\bigcup_{k=2}^{\infty}\left(A_{k} \cup B_{k} \cup C_{k}\right)\right) \cup\{x=0,0 \leqslant y<\infty\}
$$

Множество $F$ часто называют перчаткой Аракеляна. Ясно, что $\overline{\mathbb{C}} \backslash F$ связно, но не является локально связным в точке $\infty$. Согласно [15, предложение 5] для доказательства того, что $F$ есть $n$-аналитическое карлемановское множество, достаточно установить существование так называемого "подходящего исчерпания" $\left\{K_{j}\right\}_{j=1}^{\infty}$. С этой целью положим $K_{1}=\varnothing$,

$$
K_{j}=\{|x| \leqslant j,|y| \leqslant j\} \text { при } j \geqslant 2,
$$

и пусть $X_{j}=K_{j} \cup\left(K_{j+2} \cap F\right)$. Остается проверить (см. [15, равенство (10)]), что

$$
\mathscr{A}_{n}\left(X_{j}\right)=\mathscr{P}_{n}\left(X_{j}\right)
$$

При $j=1$ это следует из [13, теорема 2] и теоремы Рунге. Фиксируем теперь $j \in \mathbb{N}$, $j \geqslant 2$, и пусть $\Omega$ - жорданова область с замьканием

$$
\begin{aligned}
\bar{\Omega}= & X_{j} \backslash\left[\left(\bigcup _ { k = j + 3 } ^ { \infty } \left(\left\{x=\frac{1}{k}, k<y<\infty\right\}\right.\right.\right. \\
& \left.\left.\left.\cup\left\{x=\frac{1}{2}\left(\frac{1}{k}+\frac{1}{k-1}\right), k<y<\infty\right\}\right)\right) \cup\{x=0, y>k\}\right] .
\end{aligned}
$$

Теперь применим теорему 4 с указанным $\Omega$ и $X=K_{j}$. Поскольку в этом случае $\Omega \backslash X$ состоит из двух прямоугольников $D_{j, 1}$ и $D_{j, 2}$, причем $D_{j, i} \notin \mathscr{N}\left(\partial D_{j, i} \backslash X\right)$ при $i=1,2$ ( $\partial D_{j, i}$ имеет четыре попарно аналитически независимые стороны), имеет место равенство

$$
\mathscr{A}_{n}(X \cup \partial \Omega)=\mathscr{R}_{n}^{\bar{\Omega}}(X \cup \partial \Omega)=\mathscr{P}_{n}(X \cup \partial \Omega)
$$

Теперь, применяя теорему 3 , получаем (18), что завершает описание примера. 


\section{Список литературы}

1. Кармона Х.Х., Парамонов П.В., Федоровский К. Ю. О равномерной аппроксимации полианалитическими многочленами и задаче Дирихле для бианалитических функций // Матем. сб. 2002. Т. 193. № 10. С. 75-98.

2. Привалов И.И. Граничные свойства аналитических функций. М.-Л.: Гостехиздат, 1950.

3. Кусис П. Введение в теорию пространств $H_{p}$. М.: Мир, 1984.

4. Gauthier P. M., Hengartner W. Local harmonic majorants of functions subharmonic in the unit disc // J. Anal. Math. 1973. V. 26. P. 405-412.

5. Shapiro H. S. The Schwarz Function and its Generalization to Higher Dimensions. WileyN.Y.: Univ. of Arkansas, 1992.

6. Аракелян Н. У. Равномерная аппроксимация на замкнутых множествах целыми функциями // Изв. АН СССР. Сер. матем. 1964. Т. 28. №6. С. 1187-1206.

7. Нерсесян A. A. Равномерная и касательная аппроксимация мероморфными функциями // Изв. АН АрмССР. Сер. матем. 1972. Т. 7. С. 405-412.

8. Гайер Д. Лекции по теории аппроксимации в комплексной области. М.: Мир, 1986.

9. Gardiner S. J. Harmonic Approximation // LMS. Lecture Notes Series. V. 221. Cambridge: Cambridge Univ. Press, 1995.

10. Гамелин Т. В. Равномерные алгебры. М.: Мир, 1973.

11. Витушкин А.Г. Аналитическая емкость множеств в задачах теории приближений // УМH. 1967. T. 22. №6. C. 141-199.

12. Paramonov P. V., Verdera J. Approximation by solutions of elliptic equations on closed subsets of euclidean space // Math. Scand. 1994. V. 74. P. 249-259.

13. Carmona J. J. Mergelyan approximation theorem for rational modules // J. Approx. Theory. 1985. V. 44. P. 113-126.

14. Бuшол Э. Граничные меры аналитических дифференциалов // Некоторые вопросы теории приближений. М.: ИЛ, 1963. С. 87-100.

15. Boivin A., Gauthier P. M., Paramonov P. V. Approximation on closed sets by analytic or meromorphic solutions of elliptic equations and applications // Canad. J. Math. 2002. V. 54. № 5. P. 945-969.

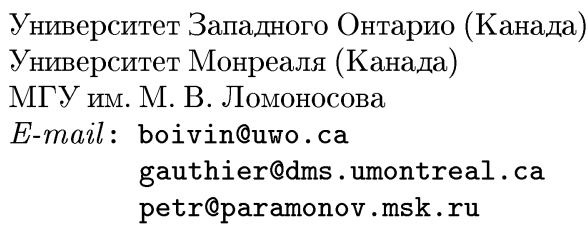

Поступило в редакцию 25.12.2003 